\title{
Biocompatibility and toxicity of graphene quantum dots for potential application in photodynamic therapy
}

\author{
Tanveer A Tabish", Chris J Scotton*,2, Daniel C J Ferguson², Liangxu Lin", Anienke van der \\ Veen ${ }^{2}$, Sophie Lowry ${ }^{2}$, Muhammad Ali ${ }^{3}$, Farhat Jabeen ${ }^{3}$, Muhammad Ali ${ }^{4}$, Paul G \\ Winyard**,2 \& Shaowei Zhang***,1 \\ ${ }^{1}$ Centre for Graphene Science, College of Engineering, Mathematics \& Physical Sciences, University of Exeter, Stocker Road, Exeter, \\ EX4 4QF UK \\ ${ }^{2}$ Institute of Biomedical \& Clinical Science, University of Exeter Medical School, St Luke's Campus, Exeter, EX1 2LU, UK \\ ${ }^{3}$ Department of Zoology, Government College University, Faisalabad, 38000, Pakistan \\ ${ }^{4}$ Faculty of Animal Sciences, Bahauddin Zakariya University, Multan, 60800, Pakistan \\ *Author for correspondence: c.j.scotton@exeter.ac.uk \\ **Author for correspondence: p.g.winyard@exeter.ac.uk \\ ***Author for correspondence: s.zhang@exeter.ac.uk
}

Aim: Achieving reliably high production of reactive oxygen species (ROS) in photodynamic therapy (PDT) remains challenging. Graphene quantum dots (GQDs) hold great promise for PDT. However, the photochemical processes leading to GQD-derived ROS generation have not yet been fully elucidated. Materials \& methods: Physicochemical characteristics of GQDs were comprehensively investigated, including electron paramagnetic resonance analysis of singlet oxygen production. Dark toxicity was assessed in vitro and in vivo. Results: GQDs demonstrated excellent photoluminescent features, corrosion resistance, high water solubility, high photo/pH-stability, in vitro and in vivo biocompatibility and very efficient singlet oxygen/ROS generation. Conclusion: The enhanced ROS generation, combined with good biocompatibility and minimal toxicity in vitro and in vivo support the potential of GQDs for future PDT application.

First draft submitted: 15 January 2018; Accepted for publication: 21 May 2018; Published online: 20 August 2018

Keywords: biocompatibility $\bullet$ corrosion resistance $\bullet$ graphene quantum dots $\bullet$ photodynamic therapy $\bullet$ photostability $\bullet$ reactive oxygen species $\bullet$ singlet oxygen $\bullet$ toxicity

Cancer accounts for over 8 million deaths annually. Standard treatment options include surgery, radio/chemotherapy and photodynamic therapy (PDT). The latter can be a particularly effective modality in the treatment of superficial tumors such as basal cell carcinoma, but has limitations due to low yields of reactive oxygen species (ROS) plus unwanted side effects. Therapeutic and diagnostic techniques based on quantum dots (QDs) are emerging as a promising paradigm for the battle against life-threatening conditions including cancer, renovascular and cardiovascular diseases, intestinal inflammatory bowel disease and neurodegenerative diseases [1-4]. The wide-ranging biological applications of QDs are due to their unique particle size, excellent photo-physical properties, luminescent features and biocompatibility [5,6]. The photoluminescence (PL) features of QDs derived from radiative recombination result in a long-lived electron-hole pair to produce a metastable state similar to the long-lived triplet state of photosensitizers (PS) and provide a cytotoxic mechanism for the production of singlet oxygen and other ROS $[7,8]$. Photo-induced electron transfer reactions of QDs have therefore recently been envisioned as potential PS for PDT. PDT is a highly selective approach to ablate tumors, without damaging surrounding tissues. Effective PDT requires three key components: a PS, light and oxygen. The combination of these three leads to the production of ROS. These highly reactive species result in a shift of redox balance and trigger the activation of transcription factors implicated in the initiation of cell death signaling via apoptosis and/or necrosis. The efficacy of PDT mainly relies on the yield of singlet oxygen and other ROS production by a PS, and this yield depends on the nature of PS involved $[9,10]$. Despite several leaps forward, a critical challenge still remains 
in relation to the inadequate oxygen supply in tumors and incapability of selectively localizing diagnostic probes and drugs at tumors sites for the purposes of early diagnosis and effective treatment $[11,12]$. One emerging strategy is to synthesize nanoplatforms, which may achieve a higher ROS yield. However, the current applications of QDs are often limited by poor water dispersibility, poor photostability, low singlet oxygen and other ROS generation yield, low biocompatibility, and a lack of imaging and therapeutic functions without attaching biomolecules or antibodies [13,14].

Graphene quantum dots (GQDs) are among the latest frontiers of research on graphene (2D monolayered carbon materials with aromatic substructure) [15]. They are typically derived from graphene/graphite or other 3D graphitic materials by various approaches and exist as mono or few-layered structures with a lateral dimension up to $100 \mathrm{~nm}$ [16]. Lower production of ROS and the intrinsic toxicity of conventional organic PS such as liposomes and polymeric nanoparticles limit their clinical applications in PDT [17]. GQDs have many advantages over conventional organic PS, such as chemical inertness, high water solubility, photostability, interplay between optoelectronic features and shape/size, good donors in the fluorescence resonance energy transfer process, high stability in physiological conditions, specific accumulation at the target site and facile surface functionalization. These features therefore make GQDs promising candidates in novel delivery systems for target-specific photosensitization [18-21] due to their PL properties [22,23], quantum confinement and edge effects [24]. Considerable efforts are being made to understand the interplay of features such as size and shape, in concert with the type and quantity of additional functional groups, for the generation of PL, as well as capacity to act as energy donors for conventional PS [16,22-24]. The energy transfer between GQDs and cell molecules, such as triplet oxygen, could potentially induce the generation of ROS, thus provoking cellular apoptosis. The light-mediated cytotoxicity of GQDs, together with their energy-donor capacity, could therefore open a new area for GQD research in the life sciences - as direct theranostics or as cofactors or components of conventional photosensitizing agents used in PDT. Recent studies have investigated alternative syntheses and PDT applicability of GQDs with encouraging results [25-27]. Although promising, GQDs will not achieve broad clinical applicability until concerns have been alleviated about toxicity from long-term exposure and quantum yield of ROS, as well as surface-dependent therapeutic responses and biological corrosion resistance.

In the present study, we report a robust, smart and highly versatile multifunctional GQD, which can be synthesized by exfoliation and disintegration from graphite flakes (GFs). We have previously reported a novel effective method to obtain water-soluble GQDs with higher yield (7.1\%), showing a size around $20 \mathrm{~nm}$, and with the majority exhibiting monolayering [28]. Crucially, we have now demonstrated limited in vivo toxicity after 4 weeks of high dose administration in rats, and subsequently confirm the enhancement of singlet oxygen and other ROS generation of GQDs. The intensity of the electron paramagnetic resonance (EPR) signal, resulting from singlet oxygen generation, showed an increase, which was dependent on the irradiation time. The ROS production from GQDs could potentially allow precise therapeutic dosing. In vivo toxicity experiments in rats demonstrate that our GQDs have minimal dark toxicity, which is a key attribute for a photosensitizer for subsequent use in PDT. In this study we also have investigated water solubility, corrosion resistance and $\mathrm{pH} / \mathrm{photostability.} \mathrm{This} \mathrm{study} \mathrm{lends}$ further credence to the theranostic potential of GQDs in disease.

\section{Experimental section}

Synthesis \& characterization of GQDs

We prepared GQDs from our previous established route by exfoliating and disintegrating GFs (also see Supplementary Note 2) [28]. Atomic force microscopy (AFM) micrographs were taken by using a VEECO Dimension 3100 Atomic Force Microscope (Bruker Scientific Instruments, MA, USA) in a tapping mode with a scan rate of $0.5 \mathrm{~Hz}$. Raman spectra were recorded on a Renishaw Raman spectrometer (Renishaw, Gloucestershire, UK) with a $532 \mathrm{~nm}$ laser beam. UV-visible (UV/Vis) spectra were recorded by a PerkinElmer Lambda 900 Spectrometer (PerkinElmer Lambda, CT, USA) at room temperature. Electrochemical behavior was examined using a CHI 660C Electrochemical Workstation ( $\mathrm{CH}$ instruments Inc., TX, USA) with a distinctive three-electrode cell: $\mathrm{Pt}$ as the auxiliary electrode, $\mathrm{Ag} / \mathrm{AgCl}$ as the reference electrode and gold as the working electrode ( $2 \mathrm{~mm}$ diameter), in a $0.1 \mathrm{M} \mathrm{KCl}$ electrolytic solution. Stability test was performed from 0.2 to $-1.0 \mathrm{~V}$ for $2 \mathrm{~h}$. Impedance measurements were carried out in the frequency range of $100 \mathrm{kHz}$ to $0.1 \mathrm{~Hz}$ at open circuit potential (OCP) with voltage amplitude of $0.005 \mathrm{~V}$. GQDs in water and heavy water solutions were dropped onto the mica substrate followed by drying at $70^{\circ} \mathrm{C}$ for $5 \mathrm{~h}$. A digital camera was used to record the images and their contact angles were calculated by PolyPro software package. FTIR spectrum of the sample was recorded in the wavenumber range of 4000-500 $\mathrm{cm}^{-1}$ using a Bruker Optics Tensor-27 FTIR spectrometer (Bruker, MA, USA). 


\section{In vitro toxicity}

Cell proliferation was evaluated with the NIH3T3 mouse fibroblast cell line (purchased from the American Type Culture Collection, VA, USA) and 16HBE14o- human bronchial epithelial cell line (obtained via Dr Jo Porter, University College London, UK). NIH3T3 cells were seeded in DMEM + 10\% FBS, 16HBEs were seeded in $\alpha$ MEM (alpha-minimum essential media) + 10\% FBS (fetal bovine serum) (Life Technologies, Loughborough, $\mathrm{UK}$ ), in 24-well plates at a density of $15 \times 10^{3}$ cells per well, and allowed to adhere overnight. Cells were then treated with GQDs diluted in $50 \mu \mathrm{l}$ tissue culture water (Life Technologies) at concentrations ranging from $1.2 \mu \mathrm{g} / \mathrm{ml}$ up to $100 \mu \mathrm{g} / \mathrm{ml}$. After $48 \mathrm{~h}$ incubation, cells were trypsinized and counted using a hemocytometer to assess cell number relative to untreated cells.

\section{In vivo toxicity}

Sprague-Dawley adult male rats (average age of 6-7 weeks, 230-250 g weight) were housed under a standard condition of a $12 \mathrm{~h}$ bright/dark sequence with free access to food. All the investigational protocols and measures were approved by the ethical committee of Government College University, Faisalabad, Pakistan. All animals were arbitrarily divided into four experimental groups (control, low dose $(5 \mathrm{mg} / \mathrm{kg})$, medium dose $(10 \mathrm{mg} / \mathrm{kg}) \mathrm{and}$ high dose $(15 \mathrm{mg} / \mathrm{kg}$ ) of GQDs), $\mathrm{n}=8$ per group. GQDs were intravenously administrated to the rats for 30 days with a 3 days pause between each injection (amounting to eight doses over 30 days). Blood samples were collected at days 1, 15 and 30, from the marginal ear vein, and used for complete blood count (CBC) and determination of selected serum biochemical parameters. Hematological parameters: red blood cells; hematocrit; mean corpuscular volume; mean corpuscular hemoglobin; hemoglobin; mean corpuscular hemoglobin concentration; platelets; lymphocytes; eosinophils (\%); monocytes (\%); white blood cells; and serum biochemical parameters: cholesterol; triglycerides; alanine transaminase; aspartate transaminase; and total protein, all analyzed in blood samples by using Hitachi 902 automatic analyser (Hitachi, Tokyo, Japan). The harvested heart, liver, lung and kidney were fixed with 4\% paraformaldehyde for $5 \mathrm{~h}$ and then dehydrated and processed for histology. $6 \mu \mathrm{m}$ sections were cut from paraffin blocks using a Reichert microtome (Reichert-Jung, NY, USA) and stained with eosin (cytoplasm staining). The stained slides were examined by light microscopy through a $20 \times$ and $40 \times$ objective lens. A histological analysis of vital organs was performed to determine whether or not the GQDs or the degradation of GQDs caused tissue damage and/or any pathologic impacts such as inflammation or necrosis.

\section{Detection of singlet oxygen \& other reactive oxygen species}

To detect ${ }^{1} \mathrm{O}_{2}$ and other ROS being produced via photochemistry by GQDs, solutions of GQDs were irradiated $\left(\lambda_{\max }=365 \mathrm{~nm}\right.$, pe300-LED) in the presence of nitroxide spin traps 4-hydroxy-2,2,6,6-tetramethylpiperidine (TMP, a ${ }^{1} \mathrm{O}_{2}$ trap, Sigma-Aldrich, Gillingham, UK), or 5-(diethoxyphosphoryl)-5-methyl-1-pyrroline- $N$-oxide (DEPMPO, an $\mathrm{O}_{2}{ }^{\bullet-}$ and ${ }^{\bullet} \mathrm{OH}$ trap, Enzo Life Sciences, Exeter, UK). Pre- and post-irradiation spectra were acquired at room temperature using an FR30 EPR spectrometer (Jeol Ltd, Welwyn Garden City, UK). Prior to irradiation, each sample was injected in to a Jeol quartz WG-LC-11 flat cell, placed into the EPR cavity and a spectrum was acquired. Samples were then irradiated for a given time, after which a second spectral acquisition was carried out. Spectral acquisition parameters for trapping of ${ }^{1} \mathrm{O}_{2}$ by TMP were: microwave frequency $9.45 \mathrm{GHz}$, microwave power $4 \mathrm{~mW}$, center field $3372 \mathrm{G}$, sweep width $50 \mathrm{G}$, sweep time $60 \mathrm{~s}$, time constant $1 \mathrm{~s}$, modulation frequency $25 \mathrm{kHz}$, modulation width $1.25 \mathrm{G}$ with an average of 3 sweeps. Spectral acquisition parameters for trapping of $\mathrm{O}_{2}{ }^{\bullet-}$ and $\bullet \mathrm{OH}$ by DEPMPO were: microwave frequency $9.45 \mathrm{GHz}$, microwave power $16 \mathrm{~mW}$, center field $3372 \mathrm{G}$, sweep width $150 \mathrm{G}$, sweep time $60 \mathrm{~s}$, time constant $1 \mathrm{~s}$, modulation frequency $25 \mathrm{kHz}$, modulation width $0.63 \mathrm{G}$ with an average of three sweeps. As a positive control for the generation and detection of ${ }^{1} \mathrm{O}_{2}$, solutions of protoporphyrin IX, a well-known photosensitizing agent, were prepared at a concentration of $10 \mu \mathrm{M}$, mixed with $50 \mathrm{mM}$ TMP and irradiated $\left(\lambda_{\max }=635 \mathrm{~nm}, 25 \mathrm{~J} / \mathrm{cm}^{2}\right.$, Aktilite CL-16). Spectra were acquired pre- and post-irradiation, and the detection of an increased EPR signal confirmed that our detection system was operating as expected. To measure the time-dependent generation of ${ }^{1} \mathrm{O}_{2}$, GQDs were prepared in $\mathrm{H}_{2} \mathrm{O}$ at a concentration of $1.3 \mathrm{mg} / \mathrm{ml}$ with $50 \mathrm{mM}$ TMP. Spectra were acquired following the irradiation of separate samples for 0, 2, 5, 10 and $30 \mathrm{~min}$, which equates to light doses of $0,0.24,0.6,1.2$ and $3.6 \mathrm{~J} / \mathrm{cm}^{2}$, respectively. The generation of ${ }^{1} \mathrm{O}_{2}$ in $\mathrm{D}_{2} \mathrm{O}$ was also measured by EPR. GQDs were prepared in $\mathrm{H}_{2} \mathrm{O}$ or $\mathrm{D}_{2} \mathrm{O}$ at a concentration of $0.5 \mathrm{mg} / \mathrm{ml}$ with $50 \mathrm{mM}$ TMP. Spectra were acquired pre and post irradiation $\left(1.8 \mathrm{~J} / \mathrm{cm}^{2}\right)$. Last, spectra of GQDs in $\mathrm{H}_{2} \mathrm{O}(0.5 \mathrm{mg} / \mathrm{ml})$ were acquired in the presence of L-histidine $(25 \mathrm{mM})$, a ${ }^{1} \mathrm{O}_{2}$ quencher $\left(1.8 \mathrm{~J} / \mathrm{cm}^{2}\right)$. The positive control for spin trapping by DEPMPO consisted of a hypoxanthine and xanthine oxidase (XO) system [29,30]. We used a solution 
(A)

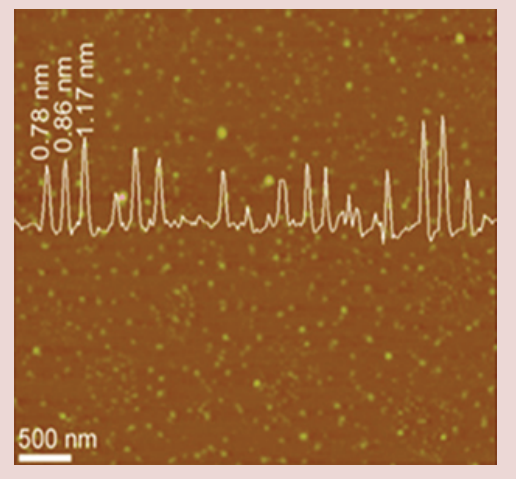

(B)

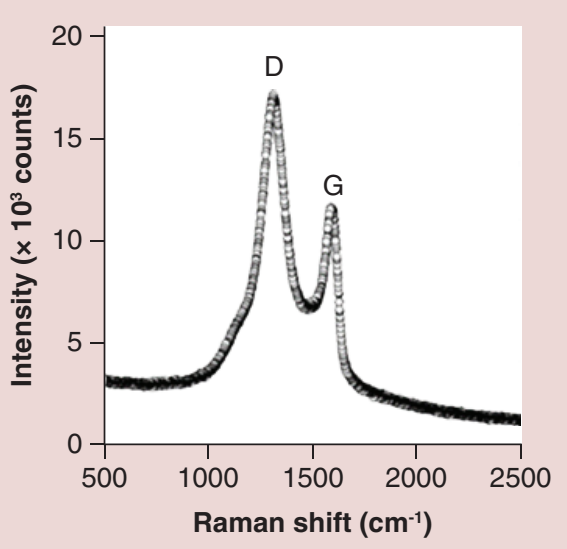

(c)

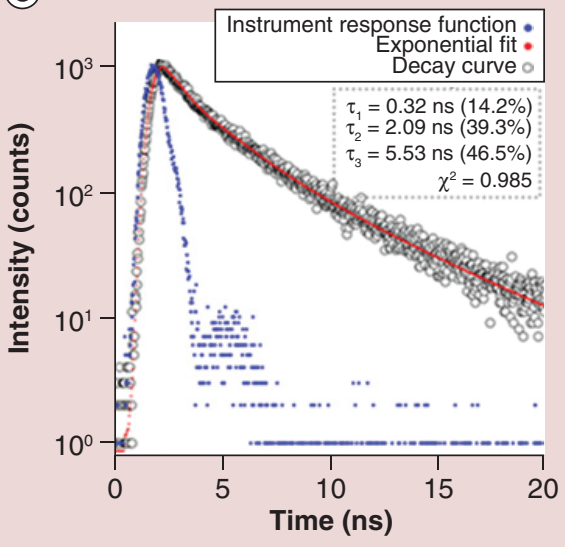

Figure 1. Basic characterization of graphene quantum dots. (A) Representative atomic force microscopy image of GQDs deposited on mica substrate indicating size around $20-40 \mathrm{~nm}$, with a particle diameter spectrum showing the complete size distribution of the GQDs (overlaid). (B) Raman spectrum demonstrating peaks for D (defect) and G (graphene) at around 1354 and $1592 \mathrm{~cm}^{-1}$, respectively. (C) time-resolved photoluminescence spectrum of the prepared GQDs, demonstrating the emission lifetime (460 nm) following GQD excitation $(360 \mathrm{~nm})$ at room temperature. GQD: Graphene quantum dot.

containing $50 \mu \mathrm{M}$ hypoxanthine, $200 \mu \mathrm{M}$ DTPA (pentetic acid), $20 \mathrm{mM}$ DEPMPO and $0.04 \mathrm{U} / \mathrm{ml} \mathrm{XO}$ in PBS (phosphate-buffered saline). The $\mathrm{pH}$ value for the PBS used in the XO system was 7.4. Following addition of XO, the solution was immediately injected into a quartz flat cell and a spectrum was acquired. GQDs $(1.3 \mathrm{mg} / \mathrm{ml})$ were mixed with DEPMPO $(20 \mathrm{mM})$ and a spectrum was acquired pre and post irradiation $\left(1.8 \mathrm{~J} / \mathrm{cm}^{2}\right)$. An additional mixture, containing GQDs, DEPMPO and DTPA $(200 \mu \mathrm{M})$ was also made and treated in the same manner.

\section{Statistical analysis}

Statistical analysis was performed between two treatment groups by unpaired Student's $t$-test, and between multiple treatment groups by one-way analysis of variance with Tukey post-hoc testing or two-way analysis of variance with Bonferroni post-hoc test, using GraphPad Prism 5 software. Results are presented as mean \pm standard deviation, unless otherwise indicated. The value of $\mathrm{p}<0.05$ was considered significant.

\section{Results}

Preparation \& basic characterization of GQDs

GQDs were prepared by following our previously established technique of exfoliating and disintegrating GFs [28]. The fundamental mechanism of the synthesis is illustrated in Supplementary Figure 1. The GQDs used herein have an AFM size around 20-40 nm (Figure 1A). AFM of the dispersed GQDs gave an average thickness of approximately $1 \mathrm{~nm}$ (Figure 1A inset), similar to the majority of other QDs due to equivalent size-dependent luminescent features of these QDs, in combination with intrinsic properties of monolayered graphene [28,31,32]. GQD crystallization was confirmed by Raman spectroscopy (Figure 1B); two characteristic D (defect) and G modes of graphene were determined at around 1354 and $1592 \mathrm{~cm}^{-1}$, respectively. The G peak of the GQDs was slightly blue-shifted from that of pure graphite and chemically reduced graphene oxide $\left(\sim 1588 \mathrm{~cm}^{-1}\right)$ [33,34], since GQDs prepared via this route have oxidized edges, making them water soluble [28]. The Fourier transform IR spectrum had two peaks: an absorption peak centered at $1637 \mathrm{~cm}^{-1}$, and a broad peak at $3402 \mathrm{~cm}^{-1}$ revealing O-H bonding (Supplementary Figure 2A). The absorptions at 1255 and $1078 \mathrm{~cm}^{-1}$ also revealed the existence of C-H and $\mathrm{C}-\mathrm{O}$, respectively. The functional groups $(\mathrm{O}-\mathrm{H}, \mathrm{C}-\mathrm{H}$ and $\mathrm{C}=\mathrm{C})$ located at the surface of the GQDs act as a passivation layer. This self-passivated surface layer facilitates the water solubility of GQDs [35,36]. The maximum luminescence peak was centered at around $460 \mathrm{~nm}$ (from 370 to $700 \mathrm{~nm}$ ) at an excitation wavelength of $360 \mathrm{~nm}$ as previously reported by us [28] with quantum yield of 7.12\% (Supplementary Table 1 and Supplementary Note 1). The time-resolved PL (time-correlated single photon counting technique) spectra of the GQDs recorded at $460 \mathrm{~nm}$ with $360 \mathrm{~nm}$ excitation laser source is shown in Figure 1C. The lifetime of the luminescence can be fitted well 
with a triexponential function: $0.32(14.2 \%$, we cannot exclude that this fast decay may arise from the instrument response), 2.09 (39.3\%) and 5.53 ns (46.5\%), respectively. The largely nanosecond decay of luminescence suggests that the GQDs are promising candidate materials for optoelectronic and biologic applications. This synthesis and basic characterization demonstrates the reproducibility of our previously reported novel fabrication route.

\section{Photostability, water dispersibility \& pH stability}

The propensity of GQDs for antibiofouling depends on the physicochemical properties of the QDs surface; key properties include the hydrophilicity of the surface and photostability. Incorporation of heavy atoms into the QDs increases the probability of intersystem crossing and also increases spin orbit coupling of an electron (known as the internal heavy-atom effect). This effect consequently increases the levels of ROS generation (see below). Water dispersibility and photostability were also measured in heavy water. The water contact angle was used to evaluate the wettability, hydrophilicity of the GQD surface and water dispersibility. The natural wettability of the surface is reflected by the contact angle parameter measured immediately through water droplets on the material surface. As shown in Figure 2A and B, the contact angle for water soluble GQDs was $11^{\circ}$, indicating the high hydrophilicity due to the existence of hydrophilic carboxylic groups [37]. The contact angle of $16^{\circ}$ for heavy water soluble GQDs was comparable to water soluble GQDs as well as $21.8^{\circ}$ on mica substrates. The hydrophilic behavior of surface amphiphilicity suggested that water and heavy water soluble GQDs both are expected to be an attractive candidate for administration in living systems. The GQDs also showed good water solubility (Supplementary Figure 3). To further investigate the effectiveness of GQDs as an optical bioimaging agent, the stability under various $\mathrm{pH}$ conditions was also verified. As shown in Figure $2 \mathrm{C}$, no obvious luminescence quenching of the corresponding PL peak $(460 \mathrm{~nm})$ was observed in various buffered solutions, demonstrating that GQDs exhibit no attenuation in PL intensity and that $\mathrm{PL}$ is largely independent of $\mathrm{pH}$, which is favorable for bioimaging applications. Photostability was also investigated over a period of $>200$ h (Figure 2D), showing that GQDs are highly photostable, which is also beneficial for biomedical applications. GQDs exhibited superior photostability compared with conventional QDs [38,39]. Overall, the good water dispersibility and solubility, effective $\mathrm{pH}$ stability and extraordinary photostability render GQDs an attractive alternative probe for efficient imaging in biological and biomedical applications.

\section{Biological corrosion resistance}

Corrosion resistance is known to play an important role in the biocompatibility assessment of bionanomaterials [40,41]. The possible release of metallic ions and particles through electrochemical corrosion phenomena induces inflammatory and toxic effects which in turn reduces the biosafety and biocompatibility of nanomaterials to solve real-world clinical problems [40,42]. Corrosion resistance depends on morphology and number of layers of QDs, exposure environment and electrochemical fluids. It is unclear whether GQDs are resistant to biological corrosion when employed in the context of bioapplications. Biological corrosion occurs in a complex aqueous environment through electrochemical processes [43], during which GQDs are oxidized to form ions which then migrate away from the GQD surface as free ions [43]. To understand these electrochemical processes, we initially measured their cyclic voltammetry (CV) curves. As shown in Figure 3A, CV curves of GQDs had high currents and good rectangular shapes. Pairs of distinctive redox peaks occurred around -8.85 (at $\left.0.098 \mathrm{~V}, 50 \mathrm{mV}^{-1} \mathrm{~s}^{-1}\right), 11.4$ (at $0.151 \mathrm{~V}, 150 \mathrm{mV} \cdot \mathrm{s}^{-1}$ ) and $-9.57 \mu \mathrm{A}$ (at $-0.171 \mathrm{~V}, 100 \mathrm{mV} . \mathrm{s}^{-1}$ ), accompanied with a small redox peak that indicated a multistep glassy carbon ion intercalation/deintercalation [44]. These high redox currents suggested a good corrosion resistance of the GQD passivation layer. They also suggested that the passivation layer on the surface of GQDs could block chemical reactions between the GQDs and body tissues during bioimaging and therapy treatment, preventing corrosion and allowing for a potentially longer implantation time. In the CV curve, there was also a small redox peak at approximately $0.1 \mathrm{~V}$ (vs Ag/AgCl), most likely arising from the multiple valence states of GQDs. Figure 3B shows the stability curve of the GQDs recorded in an electrolytic solution. The stability curve shows a negligible decrease in current density, again indicating excellent stability, even after $2 \mathrm{~h}$ of continuous operation. These characteristics of GQDs with active sites can be attributed to the high-temperature-induced strong coordination between the (QDs) surface and electrolyte, and also demonstrates their potential as a sensitive material for electrochemical sensing and bioimaging. We further used electrochemical impedance spectroscopy to monitor the interfacial properties of the self-passivated electrode. The Nyquist plot is shown in Figure 3C, revealing a high frequency of electron transfer to the GQD surface, which can be ascribed to the charge-transfer resistance $($ Rct1 $=31.70 \Omega$ ) and the double layer capacity $(\mathrm{CPE} 1=0.05, \mathrm{n}=0.542)$ occurring at the interface between the electrode and the electrolyte [45]. 
(A)
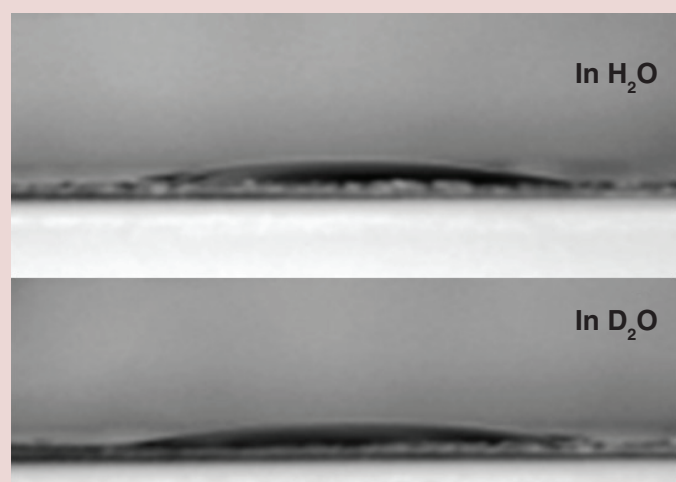

(C)

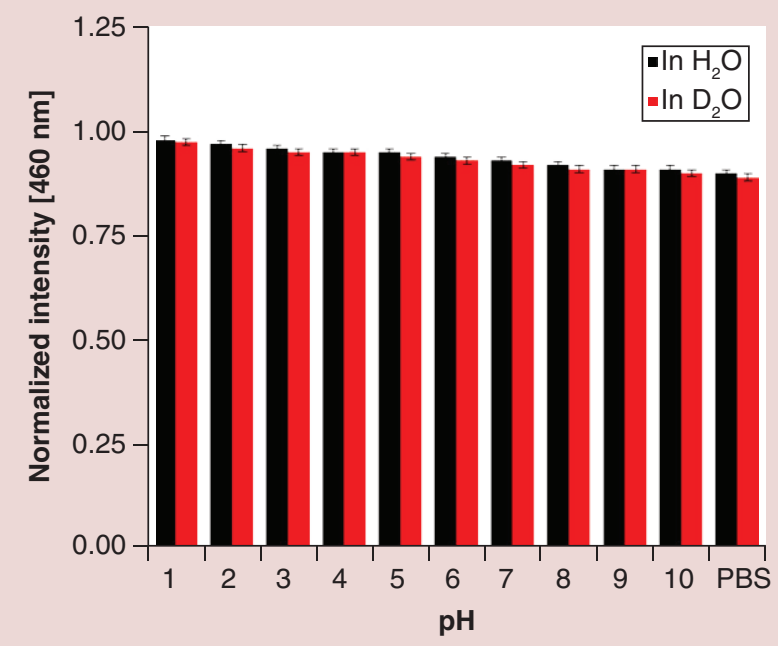

(B)

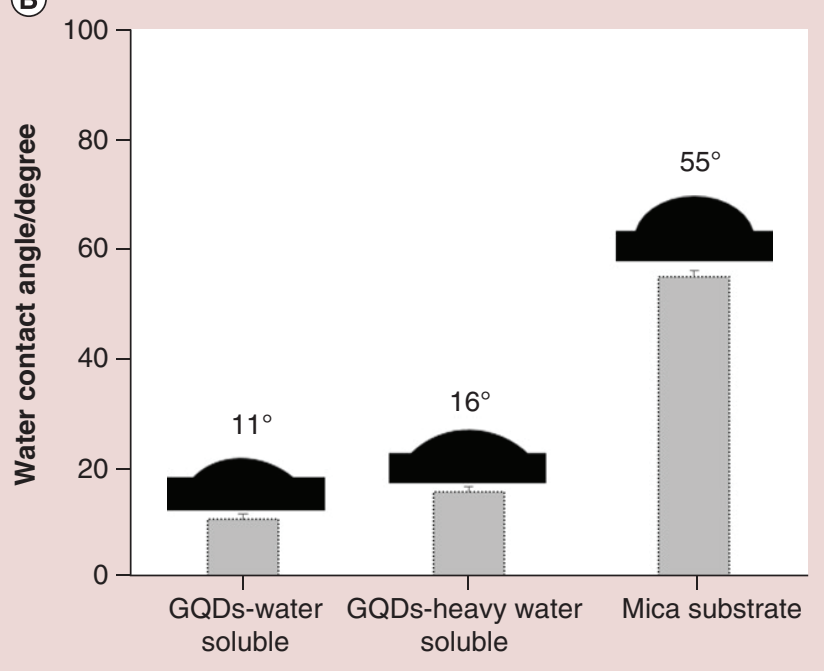

(D)

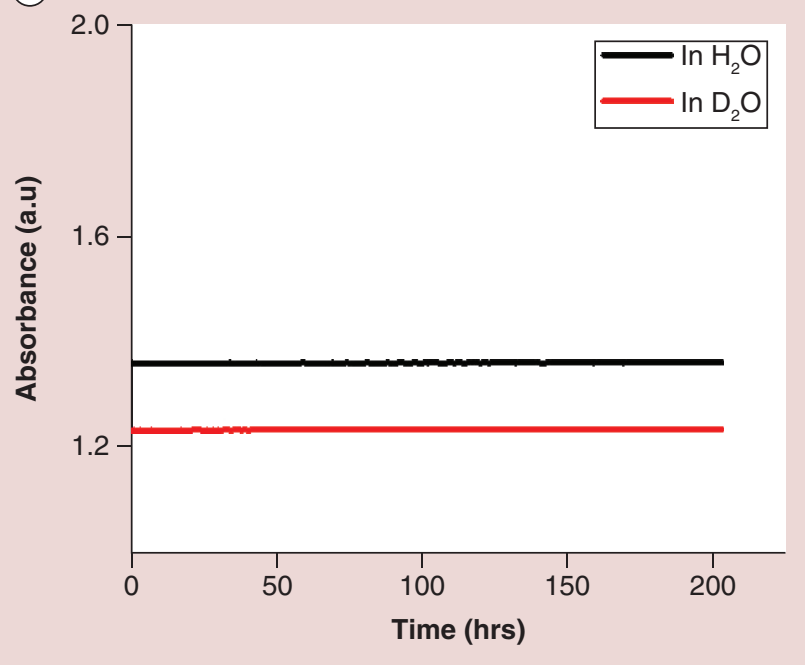

Figure 2. Water dispersibility, pH stability and photostability of graphene quantum dots. (A \& B) Representative images and quantification of wettability in $\mathrm{H}_{2} \mathrm{O}$ and $\mathrm{D}_{2} \mathrm{O}$, as measured by water contact angle, and in comparison with mica substrate; 'mica substrate' is the control without GQD. A digital camera was used to record the images and their contact angles were calculated by PolyPro software package. (C) pH-stability of GQDs in $\mathrm{H}_{2} \mathrm{O}$ and $\mathrm{D}_{2} \mathrm{O}$ indicating that no obvious luminescence quenching of the corresponding photoluminescence peak $(460 \mathrm{~nm}$ ) was observed in various buffered solutions (shown as mean \pm SD from four different experiments) and (D) Photostability of GQDs in $\mathrm{H}_{2} \mathrm{O}$ and $\mathrm{D}_{2} \mathrm{O}$ over a period of $200 \mathrm{~h}$.

GQD: Graphene quantum dot.

These results confirmed that the network of GQDs provides remarkable electronic conductivity. In this Nyquist plot, the curve at higher frequencies again indicates good corrosion resistance for biological applications [46,47]. The potentiodynamic polarization curve of GQDs was also determined (Figure 3D); the initial potential was $0.2 \mathrm{~V}$ with a corresponding current value of $-0.919 \mu \mathrm{A}$. The polarization curve obtained in this study is consistent with literature $[40,48]$ revealing that polarization plot could be divided in three potential domains: preliminary with the onset of polarization as soon as the GQDs come in contact with the saline environment; the region having the maximum value of current was labeled as partially active. There was a shift in the corrosion resistance behavior with a positive potential and current value at $-0.999 \mathrm{~V}$ and $2.6 \times 10^{-6} \mathrm{~A}$, respectively. Furthermore, it was again shifted toward corrosion inhibition at $-0.78 \mathrm{~V}$; and the formation of the passive layer started with a rise in potential and fall in the current density. This behavior demonstrated that GQDs act as a corrosion-resistant barrier because 
(A)

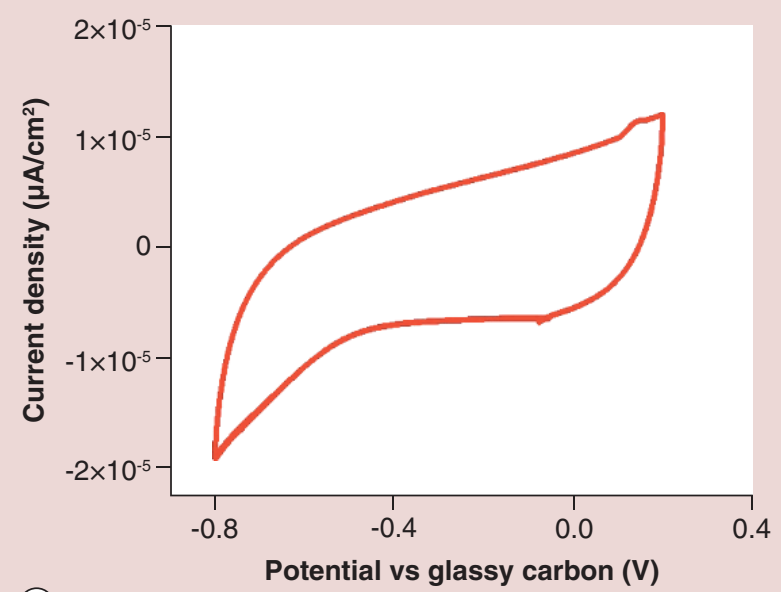

(C)

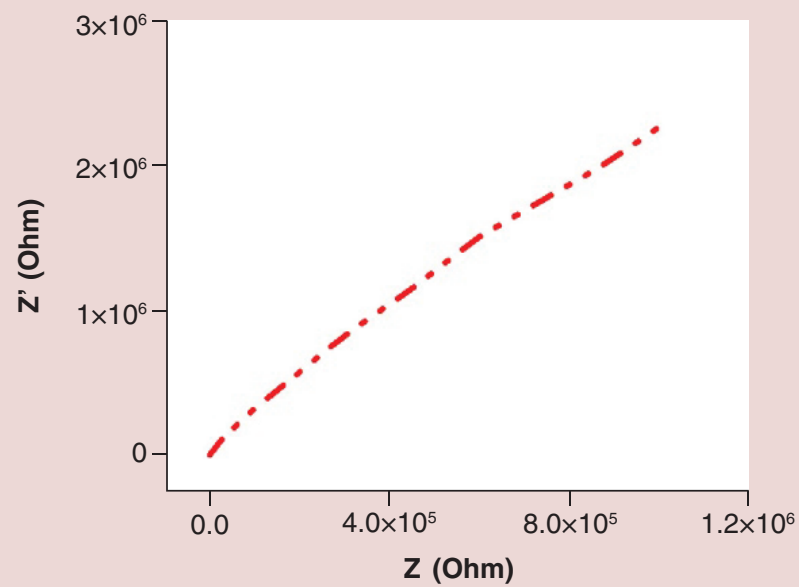

(B)

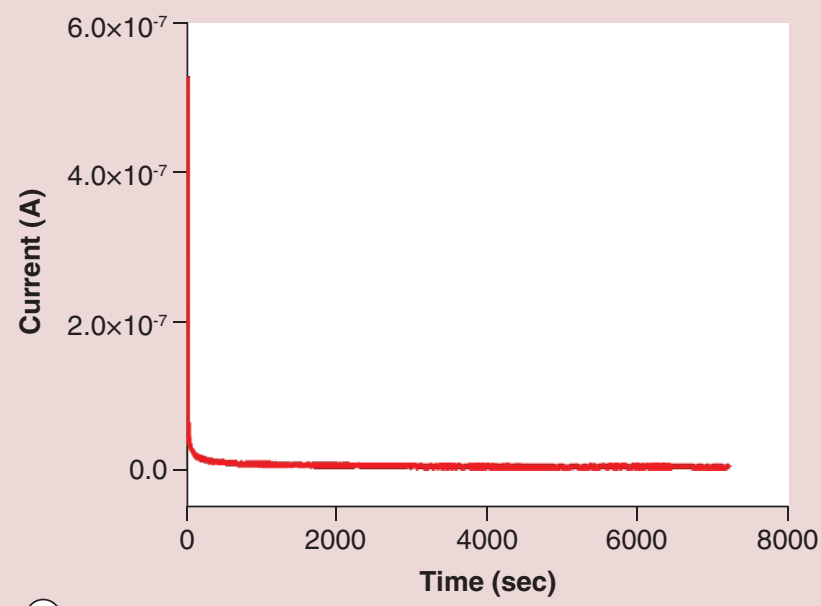

(D)

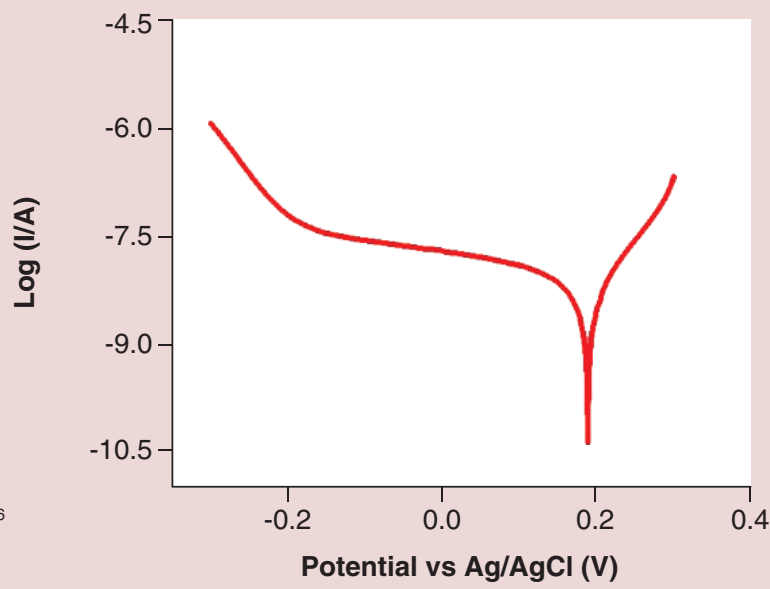

Figure 3. Electrochemical behavior of graphene quantum dot in a $0.1 \mathrm{M} \mathrm{KCl}$ electrolytic solution. (A) Cyclic voltammetry curves showing the cycle stability at scan rate of $50 \mathrm{mV}^{-1} \mathrm{~s}^{-1}$; (B) stability test performed from $0.2 \mathrm{~V}$ to $-1.0 \mathrm{~V}$; (C) electrochemical impedance spectroscopy (Nyquist plot) of graphene quantum dots. Frequency range: $100 \mathrm{kHz}-0.1 \mathrm{~Hz}$, voltage amplitude: $0.005 \mathrm{~V}$; (D) Potentiodynamic polarization curve.

a passive layer develops over a short period of time. In addition, these results suggested that the immobilized and passivated GQDs on the electrode could retain their native bioactivity [44].

\section{In vitro \& in vivo cytotoxicity}

The toxicity and biosafety of GQDs have not yet been comprehensively examined [49] and uncertainties remain about how such nanomaterials interact with biological substrates, since this is also dependent on the particular physicochemical characteristics of different formulations. In vivo toxicity will further depend on pharmacokinetic and pharmacodynamic parameters as influenced by the route of administration, dose and time period of exposure. In vitro cell viability testing using GQDs at concentrations ranging from 1.2 to $100 \mu \mathrm{g} / \mathrm{ml}$ demonstrated no obvious toxicity following a $48 \mathrm{~h}$ incubation with either a human bronchial epithelial cell line, 16HBE140 ${ }^{-}$, or murine NIH3T3 fibroblasts (Figure 4A \& B); both cell types continued to proliferate in the presence of GQDs.

To examine the in vivo cytotoxicity of GQDs, we performed a CBC, serum biochemistry and histological study of vital organs of control and treated rats in a dose-dependent manner (eight doses spread over a 30-day period of 5,10 or $15 \mathrm{mg} / \mathrm{kg}$ GQD). No clear toxic effect of GQDs on CBC was observed (Supplementary Figure 4A-L) although there was a slight $6 \%$ reduction in platelet numbers in the $10 \mathrm{mg} / \mathrm{kg}$ group at 1 week, which normalized by weeks 3 and 4; monocyte and eosinophil fractions similarly underwent a modest dose-dependent reduction, 

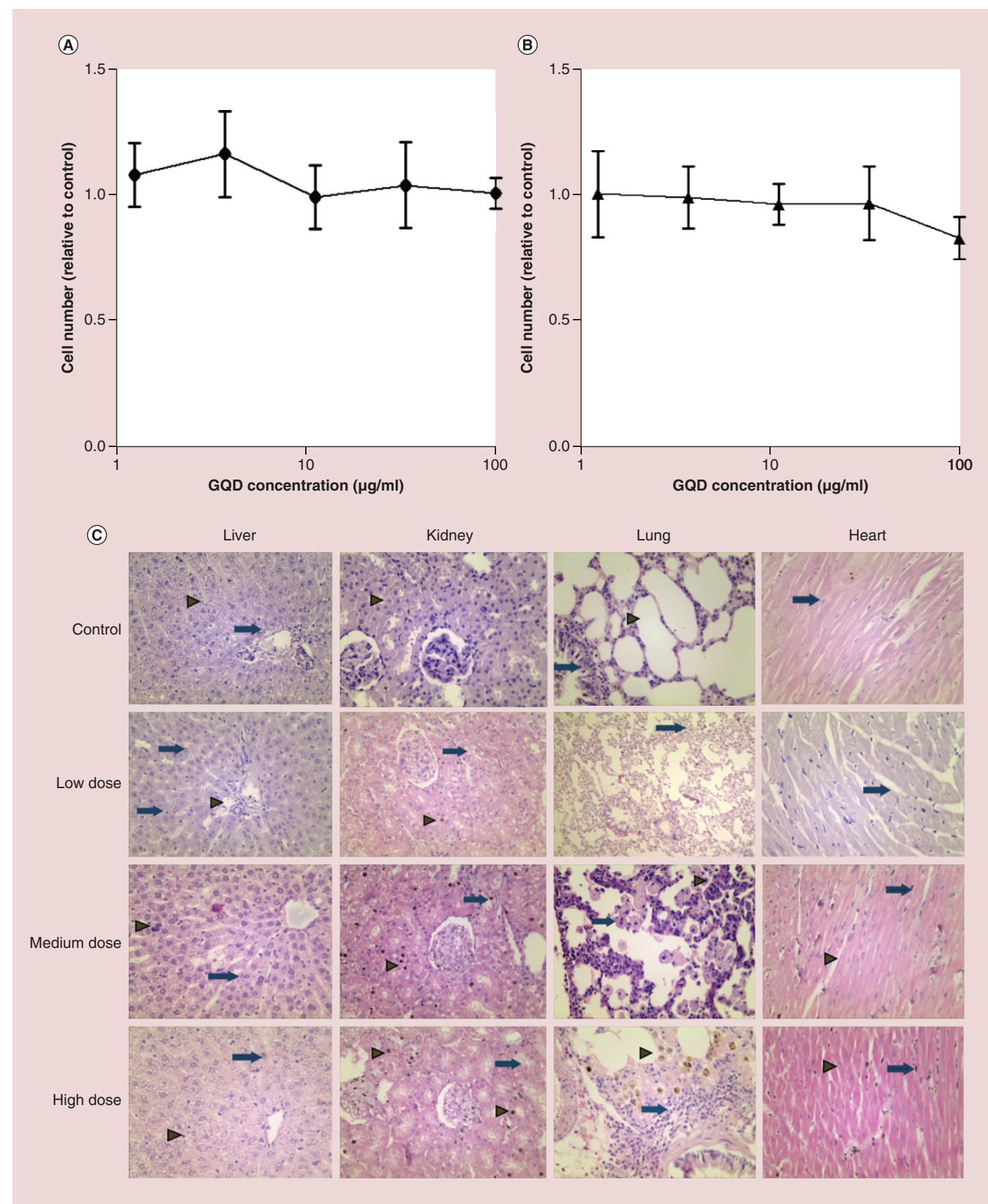

Figure 4. In vitro and in vivo toxicity of graphene quantum dots. (A) Effect of GQDs on human bronchial epithelial cell (16HBE14o-) viability after $48 \mathrm{~h}$ incubation, relative to untreated control cells. (B) Effect of GQDs on murine fibroblast (NIH3T3) viability after $48 \mathrm{~h}$ incubation, relative to untreated control cells. Data were analyzed by one-way analysis of variance with a Tukey multiple comparisons test; no significant differences were observed. (C) Histological evaluation of the vital organs of rats at 4 weeks after intravenous injection of GQDs. Tissues of low, medium and high dose of GQD-treated rats were similar to those of the control group. In the control livers, hepatocytes were arranged in cord-like pattern (arrowhead). In this section, the hepatic triad is marked as arrow. Following low-dose GQDs, Kupffer cells were evident (arrow). At medium-dose GQD, some apoptotic bodies were evident (arrowhead) and occasional binuclear hepatocytes were also seen (arrow). At the high dose of GQDs, no infiltration of polymorphic inflammatory cells was seen; Kupffer cells were still evident (arrowhead) and some apoptotic bodies were clearly seen (arrow). In the kidney, renal corpuscles are shown, with healthy renal epithelium indicated by an arrowhead. While in low-, medium- and high-dose-treated tissues, cytoplasmic vacuolation were seen in renal tubular epithelial cell (arrow) but there was no evidence of inflammatory cells in renal parenchyma. In the control lung, normal alveoli (arrowhead) and bronchioles (arrow) are shown in untreated lung tissue. Some interstitial inflammatory infiltration was seen after low-dose GQD (arrow), while giant histiocytes were present in medium-dose-treated lung parenchyma (arrow). Pigmented alveolar macrophages were present in high-dose GQD lung tissue (arrowhead). In control and low-dose heart tissue, myofibers were in a normal arrangement with healthy nuclei (arrow). There was some brown pigmentation present in the parenchyma (arrow) following medium-dose GQDs. Healthy nuclei were seen in myofibers (arrowhead) and also some brown pigmentation (arrow) following administration of high-dose GQD. Representative images shown ( $\mathrm{n}=\mathbf{8}$ rats per group), $\times 200$ original magnification. GQD: Graphene quantum dot. 
although the proportion of lymphocytes remained stable and total white cell count was unaffected. When serum biochemical parameters were compared between the groups, the results similarly did not indicate any acute toxicity. An increase in total protein was observed at 4 weeks (for all three doses), although the levels remained within the normal range (Supplementary Figure 5A-E).

A comprehensive postmortem histological study was then performed to assess any tissue interactions with GQDs. Sections of heart, kidney, liver and lung were examined for histopathological changes 30 days after GQD administration (at doses of 5, 10 and $15 \mathrm{mg} / \mathrm{kg}$ GQD). No gross abnormalities were observed, although some minor changes were particularly noted in the liver and lungs at the 10 and $15 \mathrm{mg} / \mathrm{kg}$ doses of GQDs (Figure 3B and Supplementary Tables 2 and 3 corresponding to their histological findings). No significant cardiac histology changes were evident. In the liver, some apoptotic bodies, binuclear hepatocytes and activated Kupffer cells were identified, indicating minor alterations to liver histology, but no infiltration of polymorphic inflammatory cells; hepatocyte swelling due to hepatic sinusoidal obstruction syndrome suggests some pathological effects related to GQDs. A small degree of pigmentation was observed in the kidneys of rats treated with GQDs, possibly indicating lipofuscin deposition, but again no infiltration of inflammatory cells. Within the lungs, occasional giant histiocytes were observed, associated with an inflammatory cell infiltrate, potentially indicative of a mild foreign body reaction to the GQDs. Taken together, these data indicate that GQD treatment did not result in overt acute toxicity, although the minor histopathological changes, particularly in the liver and lungs, warrant long-term observation in future studies.

\section{Singlet oxygen generation \& reactive oxygen species}

To further investigate the potential of GQDs for PDT, the singlet oxygen generation capacity of GQDs was verified and their PL in $\mathrm{H}_{2} \mathrm{O}$ and $\mathrm{D}_{2} \mathrm{O}$ was also measured (Supplementary Figure 6 shows the absorbance and PL of GQDs in $\mathrm{H}_{2} \mathrm{O}$ and $\mathrm{D}_{2} \mathrm{O}$ ). The lifetime of singlet oxygen in heavy water is ten-times longer than in water [50]; solutions were therefore prepared by adding $0.1 \mathrm{mg}$ of GQD powder into $5 \mathrm{ml}$ of $\mathrm{H}_{2} \mathrm{O}$ or $\mathrm{D}_{2} \mathrm{O}$, respectively. It was observed that the blue shift in $\mathrm{D}_{2} \mathrm{O}$ was only a little quicker than in $\mathrm{H}_{2} \mathrm{O}$ because the singlet oxygen is formed on the GQDs' surface by energy transfer from the GQDs. The $\mathrm{O}_{2}$ molecules involved in oxidation are likely to intercalate onto the surface [50]. Hence, singlet oxygen was responsible for the PL shift of the GQDs. Figure 5A shows a schematic of the mechanism for the singlet oxygen generation from a PDT photosensitizing agent. To further monitor the singlet oxygen generation, EPR spectra were recorded under different irradiation conditions and it was observed that the intensity of the signal, resulting from singlet oxygen generation, showed an increase which was dependent on the irradiation time (Figure 5B). EPR signals arising from the spin trapping of superoxide were also observed (Figure 5C). These results confirm that energy transfer from GQDs to oxygen is responsible for the excitation of ground state oxygen [51,52]. By utilizing the nitroxide spin trap, TMP, in conjunction with EPR spectrometry, we were able to demonstrate that, upon irradiation, GQDs generate ${ }^{1} \mathrm{O}_{2}$ and that this occurs in a light dose-dependent manner. TMP traps ${ }^{1} \mathrm{O}_{2}$, producing the stable radical 1-oxyl-2,2,6,6-tetramethyl-4-piperidinol (TEMPOL), which has hyperfine splitting of $\mathrm{a}^{\mathrm{N}}=16.3$. The small TMP signal observed prior to irradiation $\left(0 \mathrm{~J} / \mathrm{cm}^{2}\right)$ was attributable to low-level contamination from the manufacturing process of TMP, as this signal was also observed in solutions of TMP alone (data not shown). As the irradiation time (and thus the light dose) was increased, the concentration of ${ }^{1} \mathrm{O}_{2}$ generated increased in a linear manner $\left(\mathrm{R}^{2}=0.98\right.$, data not shown), as indicated by an increasing signal. To further demonstrate that the observed signal(s) were arising from the trapping of ${ }^{1} \mathrm{O}_{2}$ by TMP, experiments were also carried out in $\mathrm{D}_{2} \mathrm{O}$ and in the presence of the ${ }^{1} \mathrm{O}_{2}$ quencher, $\mathrm{L}$-histidine. When generated in $\mathrm{D}_{2} \mathrm{O}$, ${ }^{1} \mathrm{O}_{2}$ has a significantly longer lifetime, which was demonstrated here by a $44 \%$ increase in the concentration of the TMP reaction product detected postirradiation. In the presence of L-histidine, no appreciable EPR signal was observed, indicating complete quenching of ${ }^{1} \mathrm{O}_{2}[53,54]$. The complete lack of signal, including that arising from the previously described contamination, is likely to be due to the generation of other ROS (as described below), which are known to oxidize the product, arising from the reaction of ${ }^{1} \mathrm{O}_{2}$ with TMP, to the 'EPR silent' hydroxylamine form [55]. Thus, in the absence of any ${ }^{1} \mathrm{O}_{2}$ generation, the signal is completely eliminated. In addition, L-histidine only chemically and physically quenches/captures singlet oxygen and does not do the same with electrons. Thus the formation of the TEMPOL adduct must be caused by singlet oxygen reacting with TMP and not by electron transfer between photoexcited GQDs and TMP. If a proportion of the adduct formation was caused by electron transfer, then we would expect to see a decreased signal, not the complete absence of a signal that we observed with L-histidine. Evidence supports the view that L-histidine does not affect electron transfer [54,55]. We have also established that GQDs generate $\mathrm{O}_{2}{ }^{\bullet-}$ and ${ }^{\bullet} \mathrm{OH}$ and during irradiation. Irradiation of GQDs in the presence 

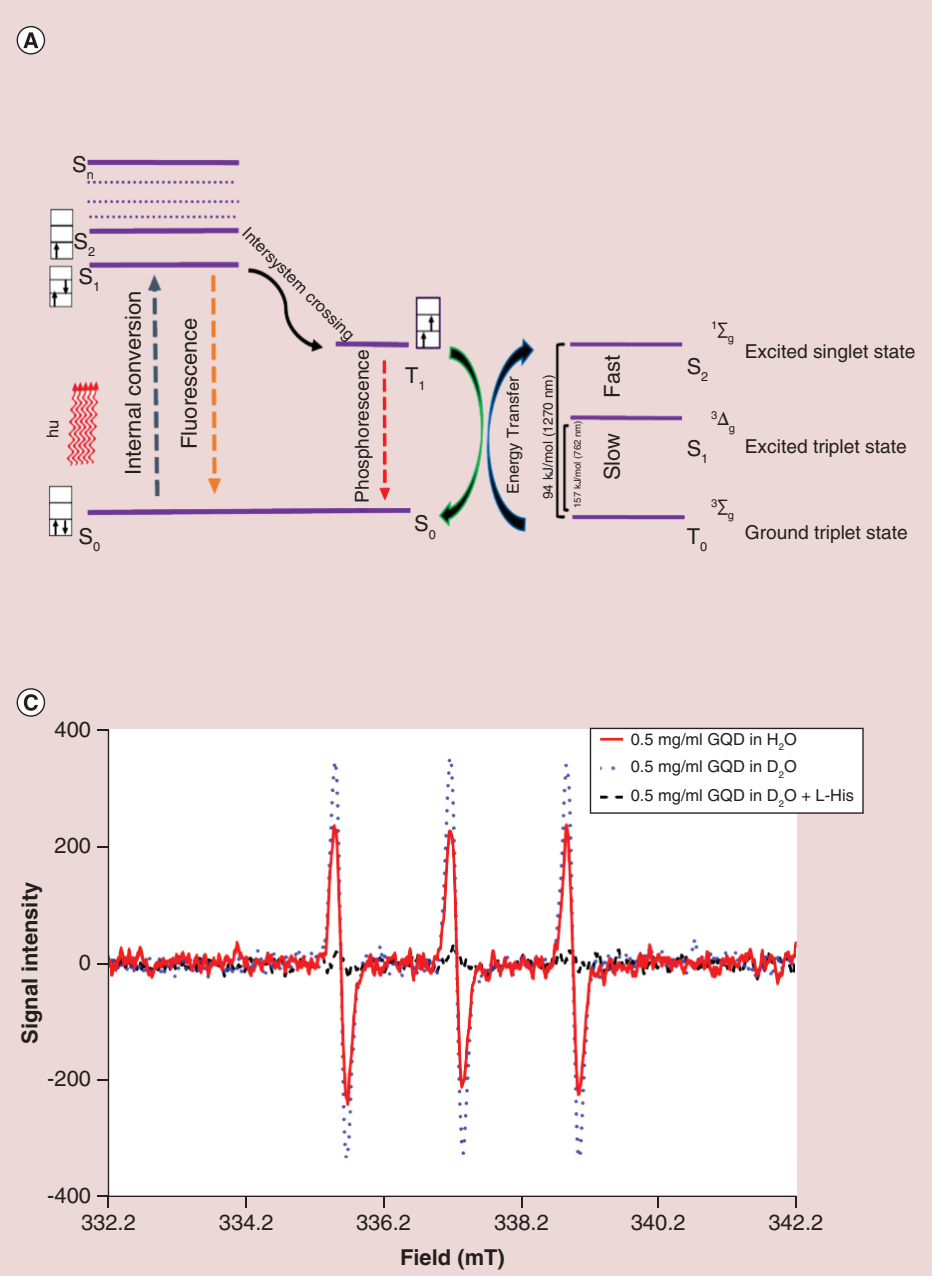

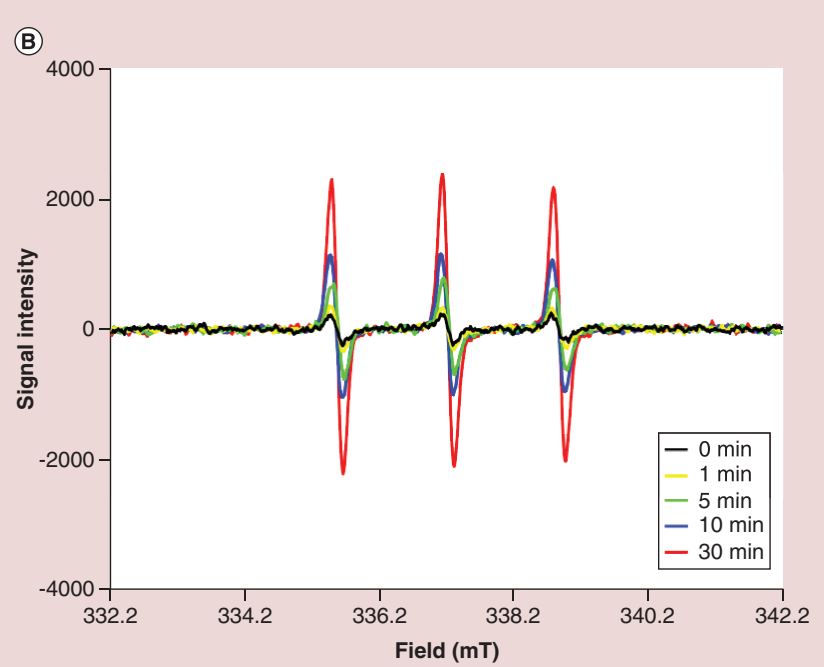

(D)

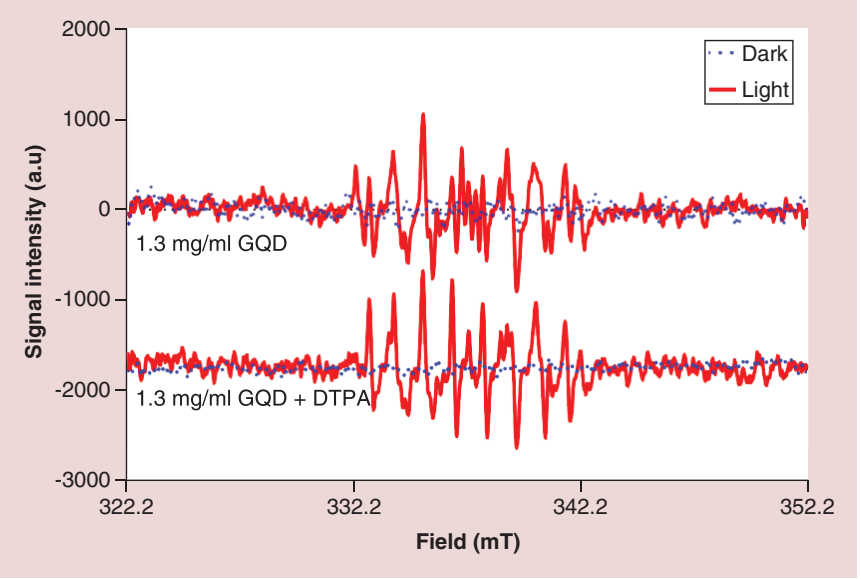

Figure 5. Singlet oxygen and reactive oxygen species generation by graphene quantum dots. (A) Schematic diagram of singlet oxygen generation through energy transfer with an excited photosensitizer. (B) Time-dependent electron paramagnetic resonance signals of GQD solutions mixed with 4-hydroxy-2,2,6,6-tetramethylpiperidine. Signal intensity increased with increasing irradiation time $\left(\lambda_{\max }=365 \mathrm{~nm}\right)$. (C) Electron paramagnetic resonance spectra following irradiation of GQDs (in $\mathrm{H}_{2} \mathrm{O}$ and $\mathrm{D}_{2} \mathrm{O}$ ) in the presence of 4-hydroxy-2,2,6,6-tetramethylpiperidine. Addition of the ${ }^{1} \mathrm{O}_{2}$ quencher, L-histidine completely attenuated the signal. (D) Electron paramagnetic resonance spectra of GQDs in DEPMPO. No signals were observed in the dark, but clear signals were observed following irradiation $\left(\lambda_{\max }=365 \mathrm{~nm}\right)$ indicating generation of ${ }^{\bullet} \mathrm{OH}$ and $\mathrm{O}_{2}{ }^{\bullet-}$. Addition of the transition metal chelator DTPA to remove ${ }^{\bullet} \mathrm{OH}$ radicals had little effect on the spectrum, indicating that $\mathrm{O}_{2}{ }^{\bullet-}$ was the dominant species.

DTPA: Pentetic acid; GQD: Graphene quantum dot.

(A): Adapted with permission from [9].

of DEPMPO resulted in an EPR signal comprised of the DEPMPO-OOH and DEPMPO-OH spin adducts $\left(\mathrm{a}^{\mathrm{N}}=13.4, \mathrm{a}^{\mathrm{P}}=52.5, \mathrm{a}^{\mathrm{H}}=11.9\right.$ and $\mathrm{a}^{\mathrm{N}}=14, \mathrm{a}^{\mathrm{P}}=47, \mathrm{a}^{\mathrm{H}}=13$; Figure $5 \mathrm{D}$, upper spectrum $)$, indicating the generation of superoxide and hydroxyl radicals, respectively. Addition of the metal chelator DTPA inhibited the generation of hydroxyl radicals [56], leaving the DEPMPO-OOH signal $\left(\mathrm{a}^{\mathrm{N}}=13.4, \mathrm{a}^{\mathrm{P}}=52.5, \mathrm{a}^{\mathrm{H}}=11.9\right.$; Figure 5D, lower spectrum).

\section{Discussion}

PDT has been regarded as a minimally invasive treatment modality and widely applied in clinics for various types of cancer treatment [57,58]. Compared with conventional therapies, PDT exhibits certain distinct advantages, since the cytotoxic photosensitizing agent can be selectively activated by manipulating the location of light exposure [59]. However, there are tremendous hurdles to overcome with standard PDT technology because of limited ROS production, lack of deep tissue penetration by the excitation wavelengths utilized, lack of selectivity toward tumor 
cells and rapid removal from the body. Assembly of luminescent graphene-related materials properties ideal for in vivo optical imaging and PDT is therefore a highly potential prospect $[60,61]$. Recent advances in the GQD field have provided unique optical properties, plus remotely controlled release of therapeutic agents in vitro and in vivo $[25,26,62]$. The use of any precursor as carbon source and their chemical processing to isolate GQDs, their size tunability and fluorescence quenching can also have a significant effect on ROS generation ability. For example, GQDs prepared from graphene oxide are actually graphene oxide QDs and they have different functional groups (such as carbonyl, carboxylic or hydroxyl groups) as compared with pristine GQDs, which in turn affect the ROS generation and toxicity of GQDs in living systems. Surface functional groups are an especially important parameter in controlling the biological activities of these QDs [9,28,63,64]. In general, several synthesis techniques are available and have been widely used for the preparation of GQDs, such as lithography [65,66], hydrothermal cutting [65,66] and electrochemical preparation [67]. The drawbacks of these methods are the low production yield and the problems associated with the separation and purification of the condensed amorphous carbon phase from low crystalline carbon blacks and fibers $[28,49,67,49,68]$. The resultant product of this partial separation and purification is an oxidized graphite framework and graphene oxide QDs rather than GQDs [65]. Efficient large-scale and cost-effective production of GQDs remains an important challenge. The features of the different synthetic methods used for the preparation of GQDs have been compared in Supplementary Table 4. Our study demonstrates a novel and facile approach to GQD fabrication through the exfoliation and disintegration of GFs, resulting in GQDs with an excellent repertoire of physicochemical properties, including biocompatibility, water dispersibility, plus high photo and $\mathrm{pH}$ stability with higher quantum yield of $7.12 \%$, showing a size of around $20 \mathrm{~nm}$, and the majority of them are single layered. Further investigation shows that these GQDs exhibit strong luminescence with high quantum yield, which is highly desirable for the real-world application of GQDs. Because of the carbene structures in the zigzag planes, the obtained GQDs exhibit exciting luminescence with high quantum yield. On the basis of these fascinating features, GQDs may be optimized for cytocompatibility and surface bioactivity, leading to unique biofunctionality not achieved through existing synthesis approaches. Crucially, they have ideal luminescent features and can generate high yields of singlet oxygen (and other oxygen radicals), above and beyond those currently achievable with state-of-the-art PDT agents. The quantum yield of our GQD preparation compares favorably with that shown in a recent study by Ge et al. [25] in which the authors use an alternative strategy for GQD preparation, based on a hydrothermal method using polythiophene derivatives. These two contrasting approaches to GQD fabrication result in nanoparticles with comparable physicochemical properties, indicating that GQDs can be robustly and easily produced with manufacturing processes which could be upscaled for future therapeutic applications while conforming to GMP guidelines. These complementary studies demonstrate that GQDs are therefore highly efficacious for PDT targeting of both cervical carcinoma xenografts in rats and breast cancer xenografts in mice [25], indicating the potentially wide applicability of this technology in a range of tumor types. Building on previous studies, we also investigated the inherent toxicity of GQDs, which is a pivotal consideration for future therapeutic application. Encouragingly, our data indicate that GQDs have minimal toxicity in fibroblasts or epithelial cells cultured in vitro. GQDs were then injected at a range of doses into healthy rats, which were monitored over a 4 -week period. The rats had no overt signs of discomfort or ill health. Post-mortem analysis revealed only limited histological changes in the liver and lungs, suggestive of a mild inflammatory reaction, but no notable changes were observed in the heart or kidneys. These findings support the biocompatibility of GQDs and alleviate concerns about the toxic side-effects of this treatment approach, although future studies should address long-term toxicology. This investigation also demonstrated that the intensity of the EPR signal, resulting from singlet oxygen generation, showed an increase which was dependent on the irradiation time. Singlet oxygen generation was also carried out in $\mathrm{D}_{2} \mathrm{O}$ and in the presence of the ${ }^{1} \mathrm{O}_{2}$ quencher, L-histidine. When generated in $\mathrm{D}_{2} \mathrm{O},{ }^{1} \mathrm{O}_{2}$ has a significantly longer lifetime, which was demonstrated here by a $44 \%$ increase in the concentration of TEMPOL detected postirradiation and the addition of the transition metal chelator DTPA inhibited the production of ${ }^{\bullet} \mathrm{OH}$, leaving an $\mathrm{O}_{2}{ }^{\bullet}$-specific EPR spectrum.

In summary, we have demonstrated a straightforward and facile approach for GQD manufacture, generating GQDs with high singlet oxygen yield, high biocompatibility and corrosion resistance, plus the high photo/pHstability, indicating their potential as a flexible, multifunctional nanoplatform for cancer therapy. The applications of GQDs are wide ranging and could be easily extended into other unique functional materials and open new doors for precision nanomedicine in future clinical applications. 


\section{Conclusion}

We herein report the enhanced yield of singlet oxygen and associated ROS from a newly synthesized GQD with potential as a smart and promising PDT agent. These GF-derived GQDs demonstrated excellent photoluminescent features, particle diameter, excellent corrosion resistance, high water solubility, high photo/pH-stability, good in vitro and in vivo biocompatibility and very efficient singlet oxygen/ROS generation. This investigation also demonstrated that the intensity of the EPR signal, resulting from singlet oxygen generation, showed an increase which was dependent on the irradiation time.

\section{Summary points}

- Our work provides a distinctive, significant, straightforward and facile approach for GQD manufacture. Crucially, we have gone on to investigate the in vivo toxicity of these GQDs following four weeks of high dose administration in rats, demonstrating excellent biocompatibility and limited toxicity, which are paramount for eventual therapeutic applications.

- Our hope is that GQDs will hold promise for use in PDT of cancer - which is entirely dependent on their ability to generate high levels of reaction oxygen species (ROS). We herein demonstrate that our GQDs have an impressive enhancement of singlet oxygen and other ROS generation over conventional photosensitizers.

- We have furthermore investigated water solubility, corrosion resistance and $\mathrm{pH} /$ photo-stability, all properties which are vital for in vivo application.

\section{Financial \& competing interests disclosure}

This work was supported by the Engineering and Physical Sciences Research Council (EPSRC) Centre for Doctoral Training in Metamaterials, XM2 (grant number EP/L015331/1), the University of Exeter, EX4 4QF, UK. The authors have no other relevant affiliations or financial involvement with any organization or entity with a financial interest in or financial conflict with the subject matter or materials discussed in the manuscript apart from those disclosed.

No writing assistance was utilized in the production of this manuscript.

Ethical conduct of research

The authors state that they have obtained appropriate institutional review board approval or have followed the principles outlined in the Declaration of Helsinki for all human or animal experimental investigations. In addition, for investigations involving human subjects, informed consent has been obtained from the participants involved.

Supplementary data

To view the supplementary data that accompany this paper please visit the journal website at: www.futuremedicine.com/doi/full/10.2217/nnm-2018-0018

Open access

This work is licensed under the Creative Commons Attribution 4.0 License. To view a copy of this license, visit http://creativecommons.org/licenses/by/4.0/

\section{References}

Papers of special note have been highlighted as: $\bullet$ of interest; $\bullet \bullet$ of considerable interest

1. Choi HS et al. Renal clearance of quantum dots. Nat. Biotechnol. 25, 1165-1170 (2007).

-. An important study on intravenous administration of quantum dots in rodents and their ultimate renal clearance, filtration and urinary excretion of such nanodots.

2. Yaghini E, Seifalian AM, MacRobert AJ. Quantum dots and their potential biomedical applications in photosensitization for photodynamic therapy. Nanomedicine 353-363 (2009).

-• Presents the potential usefulness of quantum dots as photosensitizers in photodynamic therapy compared with traditional agents such as organic dyes, which have low yield of generation of cytotoxic reactive oxygen species.

3. Samir TM, Mansour MM, Kazmierczak SC, Azzazy HM. Quantum dots: heralding a brighter future for clinical diagnostics. Nanomedicine 7(11), 1755-1769 (2012).

-. Elucidates the unique features of quantum dots in diagnosis and treatment of different diseases and their clinical challenges to solve real-world problems. 
4. Bakalova R, Ohba H, Zhelev Z, Ishikawa M, Baba Y. Quantum dots as photosensitizers? Nat. Biotechnol. 22, 1360-1361 (2004).

-• Covers the challenges in developing the next generation of photosensitizers for use in photodynamic therapy.

5. Cai W, Dong-Woon S, Kai Chen et al. Peptide-labeled near-infrared quantum dots for imaging tumor vasculature in living subjects. Nano Lett. 6, 669-676 (2006).

- Reports the preparation of arginine-glycine-aspartic acid peptide-labeled quantum dots for use in vivo imaging of tumor vasculature in athymic nude mice following subcutaneous U87MG human glioblastoma tumors and intravenous administration of quantum dots.

6. He XP, Tian H. Photoluminescence architectures for disease diagnosis: from graphene to thin-layer transition metal dichalcogenides and oxides. Small 12, 144-160 (2016).

- Provides key insights into the recent developments in the synthesis and use of graphene and transition metal dichalcogenides-based single-layer materials for single-target and multiplexed detection of a variety of biomarkers and theranostics owing to their low off-target toxicity and therapeutic responses.

7. Yaghini E, Pirker KF, Kay CW, Seifalian AM, MacRobert AJ. Quantification of reactive oxygen species generation by photoexcitation of pegylated quantum dots. Small 10, 5106-5115 (2014).

8. Yaghini E, Seifalian AM, MacRobert AJ. Quantum dots and their potential biomedical applications in photosensitization for photodynamic therapy. Nanomedicine4(3), 353-363 (2009).

9. Tabish TA, Zhang S, Winyard PG. Developing the next generation of graphene-based platforms for cancer therapeutics: the potential role of reactive oxygen species. Redox Biology. 34-40 (2018).

10. Tang R, Habimana-Griffin LM, Lane DD, Egbulefu C, Achilefu S. Nanophotosensitive drugs for light-based cancer therapy: what does the future hold? Nanomedicine 1101-1105 (2017).

11. Ferrari M. Cancer nanotechnology: opportunities and challenges. Nat. Rev. Cancer. 5, 161-171 (2005).

12. Ludwig JA, Weinstein JN. Biomarkers in cancer staging, prognosis and treatment selection. Nat. Rev. Cancer. 5, $845-856$ (2005).

13. Smith AM, Duan H, Mohs AM, Nie S. Bioconjugated quantum dots for in vivo molecular and cellular imaging. Adv. drug deliver. Rev. 60, 1226-1240 (2008).

14. Viana OS, Ribeiro MS, Fontes A, Santos BS. Quantum dots in photodynamic therapy. In: Redox-Active Therapeutics Springer International Publishing, Cham, Switzerland 525-539 (2016).

15. Kostarelos K, Novoselov KS. Exploring the interface of graphene and biology. Science 344(6181), 261-263 (2014).

-• Addresses the possible mechanisms of interaction of graphene nanostructures with biological systems. The authors suggest that graphene must be prepared in a biocompatible fashion with variety of their exposure and administration routes in living systems to determine their fate.

16. Kostarelos K, Novoselov KS. Graphene devices for life. Nature Nanotech. 9(10), 744-745 (2014).

17. Huynh E, Zheng G. Porphysome nanotechnology: a paradigm shift in lipid-based supramolecular structures. Nano Today 9(2), 212-222 (2014).

18. Li K, Liu W, Ni Y et al. Technical synthesis and biomedical applications of graphene quantum dots. J. Mater. Chem. B 5, 4811-4826 (2017).

-. Provides key insights into the synthesis of biocompatible graphene quantum dots for applications in nanomedicine.

19. Markovic ZM, Ristic BZ, Arsikin KM et al. Graphene quantum dots as autophagy-inducing photodynamic agents. Biomaterials 33(29), 7084-7092 (2012).

20. Tabish TA, Zhang S. Graphene QuantumDots: Syntheses, Properties and Biological Applications, Reference Module inMaterials Science and Materials Engineering. Elsevier, Oxford, UK (2016).

21. Jin SH, Kim DH, Jun GH, Hong SH, Jeon S. Tuning the photoluminescence of graphene quantum dots through the charge transfer effect of functional groups. ACS Nano 7(2), 1239-1245 (2013).

22. Zhu S, Zhang J, Tang $S$ et al. Surface chemistry routes to modulate the photoluminescence of graphene quantum dots: from fluorescence mechanism to up-conversion bioimaging applications. Adv. Funct. Mater. 22, 4732-4740 (2012).

23. Li LS, Yan X. Colloidal graphene quantum dots. J. Phys. Chem. Lett. 17, 2572-2576 (2010).

24. Kim S, Hwang SW, Kim M-K et al. Anomalous behaviors of visible luminescence from graphene quantum dots: interplay between size and shape. ACS Nano 6, 8203-8208 (2012).

25. Ge J, Lan M, Zhou B et al. A graphene quantum dot photodynamic therapy agent with high singlet oxygen generation. Nature Commun. 5, 4596 (2014).

26. Du D, Wang K, Wen Y, Li Y, Li YY. Photodynamic graphene quantum dot: reduction condition regulated photoactivity and size dependent efficacy. ACS Appl. Mater. Interfaces 8, 3287-3294 (2016).

27. Zhang D, Wen L, Huang R, Wang H, Hu X, Xing D. Mitochondrial specific photodynamic therapy by rare-earth nanoparticles mediated near-infrared graphene quantum dots. Biomaterials 153, 14-26 (2018). 
28. Lin L, Zhang S. Creating high yield water soluble luminescent graphene quantum dots via exfoliating and disintegrating carbon nanotubes and graphite flakes. Chem. Commun. 48, 10177-10179 (2012).

29. Winyard P, Lunec J, Blake D. Action of free radical generating systems upon the biological and immunological properties of caeruloplasmin. Int. J. Biochem. 16, 1276 (1984).

30. Winyard PG, Moody CJ, Jacob C. Oxidative activation of antioxidant defence. Trends Biochem. Sci. 30, 459 (2005).

31. Novoselov KS, Geim AK, Morozov SV et al. Electric field effect in atomically thin carbon films. Science 306, 666-669 (2004).

32. Li X, Wang X, Zhang L, Lee S, Dai H. Chemically derived, ultrasmooth graphene nanoribbon semiconductors. Science 319, 1229-1232 (2008).

33. Chen W, Yan L, Bangal PR. Chemical reduction of graphene oxide to graphene by sulfur-containing compounds. J. Phys. Chem. C 114, 19885-19890 (2010).

34. Lin L, Zhang S. Effective solvothermal deoxidization of graphene oxide using solid sulphur as a reducing agent. J. Mater. Chem. 22, $14385-14393$ (2012)

35. Tang L, Ji R, Cao X et al. Deep ultraviolet photoluminescence of water-soluble self-passivated graphene quantum dots. ACS Nano. 6, 5102-5110 (2012).

36. Yao X, Niu X, Ma K et al. Graphene quantum dots-capped magnetic mesoporous silica nanoparticles as a multifunctional platform for controlled drug delivery, magnetic hyperthermia, and photothermal therapy. Small doi:10.1002/smll.201602225 (2017) (Epub ahead of print).

37. Zeng Z, Yu D, He Z et al. Graphene oxide quantum dots covalently functionalized PVDF membrane with significantly-enhanced bactericidal and antibiofouling performances. Sci.rep. doi:10.1038/srep20142 (2016) (Epub ahead of print).

38. Krishna KV, Ménard-Moyon C, Verma S, Bianco A. Graphene-based nanomaterials for nanobiotechnology and biomedical applications. Nanomedicine 8(10), 1669-1688 (2013).

39. Li LL, Wu G, Yang G et al. Focusing on luminescent graphene quantum dots: current status and future perspectives. Nanoscale 5, 4015-4039 (2013).

40. Mazare A, Totea G, Burnei $\mathrm{C}$ et al. Corrosion, antibacterial activity and haemocompatibility of $\mathrm{TiO}_{2}$ nanotubes as a function of their annealing temperature. Corros. Sci. 103, 215-222 (2016).

41. Hamouda IM. Current perspectives of nanoparticles in medical and dental biomaterials. J. Biomed. Res. 26(3), 143-151 (2012).

42. Kulkarni M, Mazare A, Gongadze E et al. Titanium nanostructures for biomedical applications. Nanotechnology 26(6), 062002 (2015).

43. Zheng XT, Ananthanarayanan A, Luo KQ, Chen P. Glowing graphene quantum dots and carbon dots: properties, syntheses, and biological applications. Small 11, 1620-1636 (2015).

44. Zhang W, Lee S, McNear KL et al. Use of graphene as protection film in biological environments. Sci. rep. doi:10.1038/srep04097 (2014).

45. Roushani M, Abdi Z. Novel electrochemical sensor based on graphene quantum dots/riboflavin nanocomposite for the detection of persulfate. Sensor Actuat. B-Chem. 201, 503-510 (2014).

46. Vasilescu I, Eremia SA, Kusko M et al. Molybdenum disulphide and graphene quantum dots as electrode modifiers for laccase biosensor. Biosens. Bioelectron. 75, 232-237 (2016).

47. Poznyak SK et al. Size-dependent electrochemical behavior of thiol-capped CdTe nanocrystals in aqueous solution. J. Phys. Chem. B. 109, 1094-1100 (2005).

48. Bolat G, Izquierdo J, Gloriant T, Chelariu R, Mareci D, Souto RM. Investigation of processing effects on the corrosion resistance of Ti20Mo alloy in saline solutions. Corros. Sci. 98, 170-179 (2015).

49. Tabish TA, Lin L, Ali M et al. Investigating the bioavailability of graphene quantum dots in lung tissues via Fourier transform infrared spectroscopy. Interface Focus 8(3), 20170054 (2018).

50. Zhang Y, He J, Wang PN et al. Time-dependent photoluminescence blue shift of the quantum dots in living cells: effect of oxidation by singlet oxygen. J. Am. Chem. Soc. 128, 13396-13401 (2006).

51. Poznyak SK, Osipovich NP, Shavel A et al. Size-dependent electrochemical behavior of thiol-capped CdTe nanocrystals in aqueous solution. J. Phys. Chem. B 109, 1094-1100 (2005).

52. Castano AP, Mroz P, Hamblin MR. Photodynamic therapy and anti-tumor immunity. Nat. Rev. Cancer 6, 535-545 (2006).

53. Wilkinson F, Helman WP, Ross AB. Rate constants for the decay and reactions of the lowest electronically excited singlet state of molecular oxygen in solution. An expanded and revised compilation. J. Phys. Chem. Reference Data 24(2), 663-677 (1995).

54. Michaeli A, Feitelson J. Reactivity of singlet oxygen toward amino acids and peptides. Photochem. Photobiol. 59, 284-298 (1994).

55. Winyard P, Lunec J, Blake D. Action of free radical generating systems upon the biological and immunological properties of caeruloplasmin. Int. J. Biochem. Cell Biol. 16(12), 1273-1278 (1984).

56. Frejaville C, Karoui H, Tuccio B et al. 5-(Diethoxyphosphoryl)-5-methyl-1-pyrroline $N$-oxide: a new efficient phosphorylated nitrone for the in vitro and in vivo spin trapping of oxygen-centered radicals. J. Med. Chem. 38, 258-265 (1995). 
57. Gao X, Cui Y, Levenson RM, Chung LW, Nie S. In vivo cancer targeting and imaging with semiconductor quantum dots. Nat. Biotechnol. 22, 969-976 (2004).

58. Wang C, Tao H, Cheng L, Liu Z. Near-infrared light induced in vivo photodynamic therapy of cancer based on upconversion nanoparticles. Biomaterials 32, 6145-6154 (2011).

59. Chatterjee DK, Yong Z. Upconverting nanoparticles as nanotransducers for photodynamic therapy in cancer cells. Nanomedicine 3 , 73-82 (2008).

60. Du Y, Guo S. Chemically doped fluorescent carbon and graphene quantum dots for bioimaging, sensor, catalytic and photoelectronic applications. Nanoscale doi:10.1039/C5NR07579C (2016) (Epub ahead of print).

61. Tan X, Li Y, Li X et al. Electrochemical synthesis of small-sized red fluorescent graphene quantum dots as a bioimaging platform. Chem. Commun. 51, 2544-2546 (2015).

62. Qu D, Zheng M, Li J, Xie Z, Sun Z. Tailoring color emissions from N-doped graphene quantum dots for bioimaging applications. Light: Science \& Applications 4, 364 (2015).

63. Sanchez VC, Jachak A, Hurt RH, Kane AB. Biological interactions of graphene-family nanomaterials: an interdisciplinary review. Chem. Res. Toxicol. 25(1), 15-34 (2011).

64. Shen H, Zhang L, Liu M, Zhang Z. Biomedical applications of graphene. Theranostics 2(3), 283 (2012).

65. Liu R, Wu D, Feng X, Müllen K. Bottom-up fabrication of photoluminescent graphene quantum dots with uniform morphology. J. Am. Chem. Soc. 133, 15221-15223 (2011).

66. Stampfer C, Güttinger J, Molitor F, Graf D, Ihn T, Ensslin K. Tunable Coulomb blockade in nanostructured graphene. Appl. Phys. Lett. 92, 012102 (2008).

67. Tabish TA, Pranjol MZI, Karadag I, Horsell DW, Whatmore JL, Zhang S. Influence of luminescent graphene quantum dots on trypsin activity. Int. J. Nanomed.13, 1525 (2018).

68. Shen J, Zhu Y, Yang X, Li C. Graphene quantum dots: emergent nanolights for bioimaging, sensors, catalysis and photovoltaic devices. Chem. Commun. 48, 3686-3699 (2012). 
